\title{
Insulinoma-Associated Protein IA-2, a Vesicle Transmembrane Protein, Genetically Interacts with UNC-31/ CAPS and Affects Neurosecretion in Caenorhabditis elegans
}

\author{
Tao Cai, ${ }^{1}$ Tetsunari Fukushige, ${ }^{2}$ Abner L. Notkins, ${ }^{1}$ and Michael Krause ${ }^{2}$ \\ ${ }^{1}$ Experimental Medicine Section, Oral Infection and Immunity Branch, National Institute of Dental and Craniofacial Research, National Institutes of Health, \\ Bethesda, Maryland 20892, and ${ }^{2}$ Section of Developmental Biology, Laboratory of Molecular Biology, National Institute of Diabetes and Digestive and \\ Kidney Diseases, National Institutes of Health, Bethesda, Maryland 20892
}

IA-2 (insulinoma-associated protein 2), a major autoantigen in type 1 diabetes, is a receptor-tyrosine phosphatase-like protein associated with the membrane of secretory granules of neural and endocrine-specific cells. Loss of IA-2 activity in the mouse results in reduced insulin release and additional phenotypes, consistent with a general effect on neurosecretion and hormone release. To gain further insight into the cellular mechanisms of IA-2 function, we have studied the Caenorhabditis elegans homolog, CeIA- 2 encoded by the ida-1 gene. Using two independent putative null alleles of $i d a-1$, we demonstrate that animals lacking CeIA-2 activity are viable and exhibit subtle defects. Genetic studies of mutants in ida-1 and several genes involved in neurosecretory vesicle cargo release and signaling highlight two roles for CeIA-2. First, CeIA-2 has a specific and novel genetic interaction with UNC-31/CAPS, a protein that has been shown in other systems to regulate dense-core vesicle cargo release. Second, loss of CeIA-2 activity enhances weak alleles in the insulin-like signaling pathway. These results suggest that CeIA-2 may be an important factor in dense-core vesicle cargo release with parallels to insulin signaling in mammals.

Key words: autoantigen; diabetes; insulin; synaptic transmission; dense core vesicle; protein tyrosine phosphatase; PTP; aldicarb; dauer; C. elegans

\section{Introduction}

Vesicle trafficking in neuroendocrine cells and neurons is essential for proper hormone signaling and neurotransmission. The two prominent neurosecretory vesicle types are synaptic vesicles (SVs) and dense-core vesicles (DCVs). SVs mediate the release of fast-acting neurotransmitters such as glutamate and acetylcholine, whereas DCVs mediate the release of hormones, neuropeptides, and biogenic amine neurotransmitters. Many components involved in SV trafficking have been identified, providing a rich cellular and molecular understanding of this pathway in neurons. Several of these factors have subsequently been shown to also regulate DCV cargo release (Gerber and Sudhof, 2002). However, there is some evidence that DCV trafficking involves some components not involved in SV trafficking. For example, a calciumactivated protein for secretion (CAPS) seems to be a specific regulator of DCV fusion to the plasma membrane (Walent et al., 1992; Grishanin et al., 2002). It is important to determine the

\footnotetext{
Received July 22, 2003; revised Feb. 1, 2004; accepted Feb. 2, 2004.

Some strains used in this work were provided by the Caenorhabditis Genetics Center, which is funded by the National Institutes of Health Center for Research Resources. We thank Tommy Brodigan, Charles Wohlenberg, and Liping Feng for technical help, Dr. K. Iwasaki and Dr. M. Nonet for antibodies, Dr. C. Bargmann for a GFP reporter strain, and Andy Golden and Joan McDermott for comments on this manuscript.

Correspondence should be addressed to Dr. Michael Krause, Section of Developmental Biology, Laboratory of Molecular Biology, National Institute of Diabetes and Digestive and Kidney Diseases, National Institutes of Health, Bethesda, MD 20892. E-mail: mwkrause@helix.nih.gov.

DOI:10.1523/JNEUROSCI.0101-04.2004

Copyright $\odot 2004$ Society for Neuroscience $\quad 0270-6474 / 04 / 243115-10 \$ 15.00 / 0$
}

identity and function of additional DCV pathway components to fully understand important cellular processes, such as hormone release.

IA-2 (insulinoma-associated protein 2, also known as ICA512 ) and its paralog, IA- $2 \beta$ (phogrin), are among the components that have been implicated in DCV trafficking (Lan et al., 1994; Kawasaki et al., 1996; Lu et al., 1996). IA-2 and IA-2 $\beta$ are transmembrane protein-tyrosine phosphatase-like (PTP) proteins belonging to an evolutionarily conserved family (Cai et al., 2001). Although highly related to PTPs, IA-2 family members lack phosphatase activity because of amino acid substitutions in the catalytic domain (Magistrelli et al., 1996; Wishart and Dixon, 1998; Cai et al., 2001). These amino acid substitutions are evolutionarily conserved, suggesting that the lack of enzymatic activity is a critical feature of these proteins (Wishart and Dixon, 1998).

IA- 2 and IA- $2 \beta$ have been localized to the membrane of DCVs in neurons and peptide-secreting endocrine cells, including pancreatic $\beta$-cells (Lan et al., 1994; Solimena et al., 1996; Pouli et al., 1998). Both proteins have been shown to undergo calciumdependent phosphorylation in response to secretagogues, and IA-2 interacts with the plasma membrane cytoskeleton protein spectrin (Berghs et al., 2000; Ort et al., 2001). In IA-2-deficient mice, only $50-60 \%$ of the normal amount of insulin was secreted from isolated pancreatic islets by glucose stimulation in vitro (Saeki et al., 2002). Moreover, in IA-2-deficient mice, blood glucose levels were significantly elevated in glucose tolerance tests 
(Saeki et al., 2002). These findings suggest that IA-2 family members may play an important role in the regulated secretion of insulin. IA-2 is also a major autoantigen associated with type 1 diabetes, thereby providing diagnostic information for disease, often before the onset of clinical symptoms (Lan et al., 1996; Notkins, 2002).

We have turned to the nematode Caenorhabditis elegans as a model system to aid in our understanding of the function of IA-2 family members. Previous studies have shown that $C$. elegans contains a single gene ( $i d a-1)$ related to IA- 2 and IA- $2 \beta$, and it is expressed predominantly in the nervous system (Cai et al., 2001; Zahn et al., 2001). C. elegans is a genetically tractable system with a simple nervous system (302 neurons) that has been extensively described at the cellular and ultrastructural levels. C. elegans studies have made major contributions to our understanding of SV trafficking, providing genetic and molecular characterization of key components (Rand and Nonet, 1997; Chalfie and Jorgensen, 1998). It seems likely that $C$. elegans will serve an equally important role in understanding DCV trafficking and may provide important information on IA-2 function in mammalian neurosecretion.

As a first step in the functional analysis of the C. elegans IA-2 protein (CeIA-2), we have characterized phenotypes associated with putative null alleles of the gene ida-1. We show that animals lacking CeIA-2 function are viable but have subtle defects attributable to deficits in acetylcholine release from the presynaptic membrane. Using double-mutant combinations between ida-1 and a variety of genes involved in SV and DCV trafficking and cargo release, we provide evidence that CeIA-2 primarily interacts with factors in the DCV pathway. ida-1 has a specific and novel genetic interaction with uncoordinated mutants of the gene $u n c-31$, encoding the C. elegans homolog of CAPS, a protein that regulates DCV cargo release in mammals and Drosophila. Moreover, mutants of ida-1 preferentially enhance the dauer phenotype of weak alleles in the insulin-like signaling pathway. These results suggest that CeIA-2 is required for proper DCV cargo release with striking parallels to insulin signaling in mammals.

\section{Materials and Methods}

Strains. Strains used were as follows: wild-type N2, ida-1(ok409) III, ida-1(tm334) III, unc-18(e81) X, unc-31(e169) IV, unc-31(e928) IV, unc64(e246) III, unc-104(e1265) II, daf-2(e1370) III, daf-7(e1372) III, daf-11(m47) V, daf-12(rh273) X, daf-28(sa191) V, rab-3( js49) II, and $a e x-3(s a 5)$ X. A pair of primers ( $5^{\prime}$-CTAATCGACACTGGTCTCAC-3' and $5^{\prime}$-CGTTCCATTGAGCCTCAATC- $3^{\prime}$ ) was designed and sent to the C. elegans gene knock-out group at the Oklahoma Medical Research Foundation for screening of ida-1 mutants. A deleted allele, designated ida-1(ok409) III, was isolated and kindly provided to us. The second allele, ida-1(tm334) III, was kindly provided by Dr. Shohei Mitani’s (Tokyo Women's Medical University School of Medicine, Tokyo, Japan) laboratory. These two mutants were outcrossed with N2 a minimum of four times, and then one or both alleles were crossed into each of the following genetic backgrounds to create double mutants: unc-18(e81) X, unc31(e169) IV, unc-31(e928) IV, unc-64(e246) III, unc-104(e1265) II, daf2(e1370) III, daf-7(e1372) III, daf-11(m47) V, daf-12(rh273) X, daf-28(sa191) V, rab-3( js49) II, and aex-3(sa5) X. Double mutants were confirmed by phenotype, molecular genotyping of specific alleles, or both. For making double mutants between the linked alleles, F2 homozygotes for the visible phenotype were selected, and the presence of the ok409 or tm334 allele was determined by PCR. Double homozygotes were then selected from siblings. The ida- 1 genotype was confirmed by use of a single worm PCR (Williams, 1995) with three primers, two of which (5'-CACCGAGGATCCGACTATTC- $3^{\prime}$ and $5^{\prime}$ - TTCTTGGAGGAAGTTCTGG-3') flank the deletion break points and one (5'-GAACTCTCGGACG GTCAGCTTAAC- $\left.3^{\prime}\right)$ located within the deletion. Flanking primers yielded a PCR product of 365-bp for the $o k 409$ allele or 1543-bp for the tm334 allele; the wild-type allele yielded a 2077 bp product. The exact deletion points of $o k 409$ and tm334 were confirmed by DNA sequencing.

Locomotion assays. Standard locomotion assays were performed as previously described (Miller et al., 1999). Briefly, the locomotion rate was quantified by counting body bends of young adult animals on thin lawns of Escherichia coli strain OP50 bacteria. The assay was set up by placing single late larval stage 3 (L3) or early L4 larva on each of 10 locomotion assay plates. Plates were then placed at room temperature for $24 \mathrm{hr}$, during which time the larva matured to young adults. To count body bends, a plate was placed on the dissecting microscope for $30 \mathrm{sec}$, and then body bends were counted for $3 \mathrm{~min}$. A body bend was counted each time the tip of the tail passed through the maximum or minimum amplitude. After all 10 plates had been counted, the process was repeated two more times on the same set of animals. Mean body bends per minute were calculated for each animal, and these means were used to calculate the mean and SE of the entire set of 10.

Dauer formation. Adults were allowed to lay eggs on plates for $3 \mathrm{hr}$ at room temperature, and progeny were scored after $48 \mathrm{hr}$ at $26^{\circ} \mathrm{C}$ (Ailion and Thomas, 2000). Dauers were distinguished by the distinctive body shape, darkly pigmented intestine, and a constricted pharynx. Partial dauers, which were included in the dauer category for scoring, lacked at least one of these characteristics. Incubation temperature is a critical variable in dauer assays. Each set of assays included all of the relevant strains, and the actual ambient incubator temperature surrounding the plates was monitored with a digital thermometer (Barnant Co., Barrington, IL).

Pharmacological assays. Acute effects of aldicarb and levamisole on mutants were assessed in a paralysis assay as described (Nonet et al., 1993). Sensitivity to aldicarb was examined by transferring 50 adult animals to plates containing aldicarb and assaying the time course of paralysis. Animals were considered paralyzed if they appeared hypercontracted and failed to move even if prodded with a platinum wire. Chronic effects of aldicarb on mutants were quantified in a growth assay as previously described (Nguyen et al., 1995; Miller et al., 1999). Single L1 larva were placed on individual culture plates containing $0,10,25,50,75,100$, 200,300 , or $400 \mu \mathrm{M}$ aldicarb and grown at room temperature $\left(\sim 22^{\circ} \mathrm{C}\right)$ for $96 \mathrm{hr}$. Growth was then stopped by putting the plates at $4^{\circ} \mathrm{C}$. For each concentration, the number of progeny was counted as a percentage of the number of progeny produced on the no-drug control. Triplicate assays were performed, and an N2 control set was included with each set of strains to allow comparisons between different sets of assays.

Transgenic lines. Wild-type C. elegans received injections of plasmid constructs or cosmid DNA at concentrations of $50-100 \mu \mathrm{g} / \mathrm{ml}$ along with $50 \mu \mathrm{g} / \mathrm{ml} \mathrm{pRF4} \mathrm{rol-6(d)} \mathrm{as} \mathrm{a} \mathrm{transformation} \mathrm{marker} \mathrm{(Mello} \mathrm{and} \mathrm{Fire,}$ 1995); pRF4 was injected alone at $50 \mu \mathrm{g} / \mathrm{ml}$ as a control for Roller effects. At a minimum, two stably transformed strains were established for each test construct. A CeIA-2::green fluorescent protein (GFP) translational fusion construct in the pPD95.69 vector was driven by a 3282-bp 5' upstream sequence of CeIA-2 as described before (Cai et al., 2001). An integrated line of the CeIA-2::GFP reporter construct, designated KM246, was generated by irradiation of one of the stable extrachromosomal array strains (Egan et al., 1995). The integrated line was backcrossed two times to lessen the frequency of second site mutations caused by the irradiation used for integration. The CeIA-2::GFP cDNA was also driven by a 3265 bp 5 ' upstream sequence of acr-2 (acetylcholine receptor subunit) for expression of the CeIA-2::GFP fusion protein in VA, DA, VB, DB, DA, IL1, RMD, and PVQ motor neurons (Squire et al., 1995; Nurrish et al., 1999). The acr-2 promoter was PCR-amplified with primers (5'-CAGTTGACTGCAGAAGTCAGAGCTGTGGAAGTCG-3' and 5'-GACCGTCTTGGCCATGAAAACGGCGTCCTTCCTG-3') and confirmed by sequencing.

Antibody staining. Immunocytochemistry was performed using a modification of the protocol of Finney and Ruvkun (1990), as described by Nonet et al. (1993). Antisera against synaptotagmin (antibody 1095; kindly provided by M. Nonet, Washington University School of Medicine, St. Louis, MO) and RAB-3 (kindly provided by K. Iwasaki, National Institute of Bioscience and Human Technology, Ibaraki, Japan) were used as described (Iwasaki and Toyonaga, 2000). Antisera against sero- 
tonin were obtained from H. Steinbusch (Maastricht University, Maastricht, the Netherlands) and performed using a whole-mount procedure as described in a detailed protocol (http://home.sandiego.edu/ cloer/ loerlab/anti5htshort.html; Loer and Kenyon, 1993). Anti-GFP antibodies were obtained from Clontech (Palo Alto, CA). Antibodies were detected using rhodamine- or FITC-conjugated donkey anti-rabbit or goat anti-mouse secondary antibodies (Jackson ImmunoResearch, West Grove, PA).

\section{Results}

\section{A punctate axonal pattern of CeIA-2::GFP colocalizes} with synaptotagmin

Previous studies of ida- 1 expression relied on transcriptional fusions with a GFP reporter gene (Cai et al., 2001; Zahn et al., 2001). Expression was almost exclusively seen in neurons, although the number of expressing neurons varied depending on the promoter element used. A $3.3 \mathrm{~kb}$ upstream promoter element gave widespread expression, with most or all neurons showing some level of expression (Cai et al., 2001). A $7.6 \mathrm{~kb}$ upstream promoter element gave a more restricted pattern of expression limited to $\sim 30$ neurons (Zahn et al., 2001).

To address the subcellular distribution of IA-2, we generated a translational fusion between the complete CeIA-2 cDNA and GFP driven by a $3.3 \mathrm{~kb}$ upstream promoter region of $i d a-1$. Multiple independent transgenic strains showed very similar patterns of expression; CeIA-2::GFP was easily detected in a restricted set of $\sim 13$ neurons. Most of these neurons were a subset of those reported by Zahn et al. (2001). CeIA-2::GFP was strongest in the ALA neuron and easily detectable in the VCs, HSNs, and PHCs. A lower level of GFP was also detected in the uv1 secretory cells (Fig. 1 ). Some, but not all, of these expressing neurons have previously been shown by electron microscopic studies to contain DCVs (White et al., 1986). However, not all DCV-containing neurons expressed this GFP reporter gene. We believe that the pattern of GFP expression from this reporter gene likely under-represents the normal CeIA-2 distribution because of previous transcriptional reporter results and very faint GFP that can be observed in additional neurons under certain conditions (see below).

To decrease the level of mosaic expression typically observed for extrachromosomal transgenes, and for further genetic studies, we generated an integrated transgenic strain $\left(\mathrm{P}_{\text {ida-1 }}\right.$ CeIA-2::GFP, KM246), harboring this reporter gene. The expression pattern of the integrated strain was similar to that seen with extrachromosomal arrays. Despite integration, there was still a degree of mosaic expression, most notably in the VC motor neurons.

A striking aspect of the ida-1 translational GFP reporter expression was the subcellular distribution of GFP. $\mathrm{P}_{i d a-1} \mathrm{CeIA}-2:: \mathrm{GFP}$ was observed in neuronal cell bodies and in puncta along the length of axons of neurons in which it was expressed (Fig. 1). A punctate distribution of other neuronal components, such as synaptobrevin and synaptotagmin, has been shown to reflect accumulation of fusion protein-tagged vesicles at synapses (Nonet, 1999). To determine whether the $\mathrm{P}_{\text {ida-1 }}$ CeIA-2::GFP axonal pattern was coincident with synapses, we stained animals expressing this CeIA-2::GFP transgene with an antibody against synaptotagmin. As shown in Figure $1 B$, the punctate GFP reporter pattern in axons colocalized with synaptotagmin staining, suggesting clustering of CeIA-2::GFP to synaptic regions.

\section{Characterization of two putative null ida-1 alleles}

We obtained two deletion alleles of ida-1(ok409 and tm334) to study the effect of loss of CeIA-2 activity in C. elegans. We sequenced PCR products amplified from both mutant animals to characterize the nature of each deletion (Fig. 2). The $o k 409$ allele

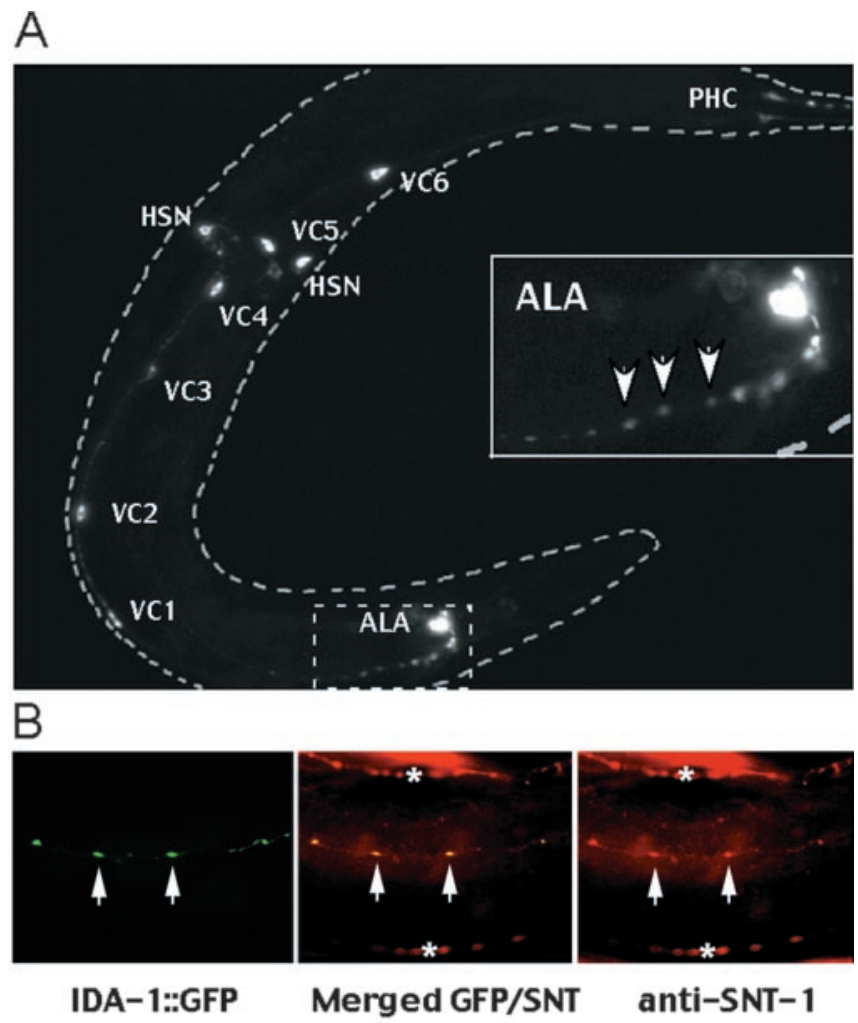

Figure 1. CelA-2 GFP reporter gene expression. $A$, GFP fluorescence image of a transgenic animal harboring the $\mathrm{P}_{\text {ida-7 }}$ CelA-2::GFP reporter gene. Very strong expression is detected in the ALA, VC, HSN, and PHC neurons as labeled. Lower-level expression is seen in the uv1 neurosecretory cells of the vulva. Dashed lines indicate the outline of the animal, with the head at the bottom and the tail in the top right corner. The punctate pattern of the $\mathrm{P}_{\text {ida- } 1}$ CelA-2::GFP reporter in axons is most easily observed in the solitary lateral axons of $A L A$, one of which is visible in the focal plane shown. The boxed region of the head expression pattern of the ALA neuron is shown at a higher magnification in the inset. Arrowheads indicate the punctate pattern of GFP observed in most axons of GFP-positive neurons. B, Coincidence of CelA-2::GFP puncta and Synaptotagmin-labeled synapses. Puncta (arrows) in a lateral ALA axon segment just posterior to the pharynx are shown. These puncta are visualized by CelA-2::GFP expression (far left) and are revealed as synapses by anti-synaptotagmin antibody staining (far right). The center panel merges the two outside images with anti-synaptotagmin in red and CelA-2::GFP in green with overlap in yellow. Asterisks mark the ventral and dorsal nerve cords that are slightly out of the focal plane and that are heavily labeled by anti-synaptotagmin staining.

resulted from a 1713 bp deletion with break points in introns 3 and 7. The deleted ida-1(ok409) gene would encode a protein product from only exons 1-3 because of a frame shift and stop codon resulting from the predicted mRNA splicing. The $t m 334$ allele resulted from a 534 bp deletion with break points in exons 3 and 4 (Fig. 2). The deleted ida-1(tm334) gene would encode a protein from only exons 1,2 , and most of 3 because of a frame shift and stop codon two amino acids downstream of the deletion break point. We have used reverse transcription-PCR to confirm the predicted mRNA products and premature stop codons for each mutant. Both deletion alleles are predicted to encode similarly truncated CeIA-2 proteins that lack the C-terminal twothirds of the protein, including the transmembrane domain and the protein-tyrosine phosphatase-like domain. Both ida-1 alleles are assumed to be nulls based on the molecular characterization.

Loss of CeIA-2 activity results in subtle visible phenotypes Animals homozygous for ida-1(ok409) or ida-1(tm334) are viable and fertile. Assays for rate of development and chemotaxis appeared normal in the homozygous mutants. Homozygous ida- 


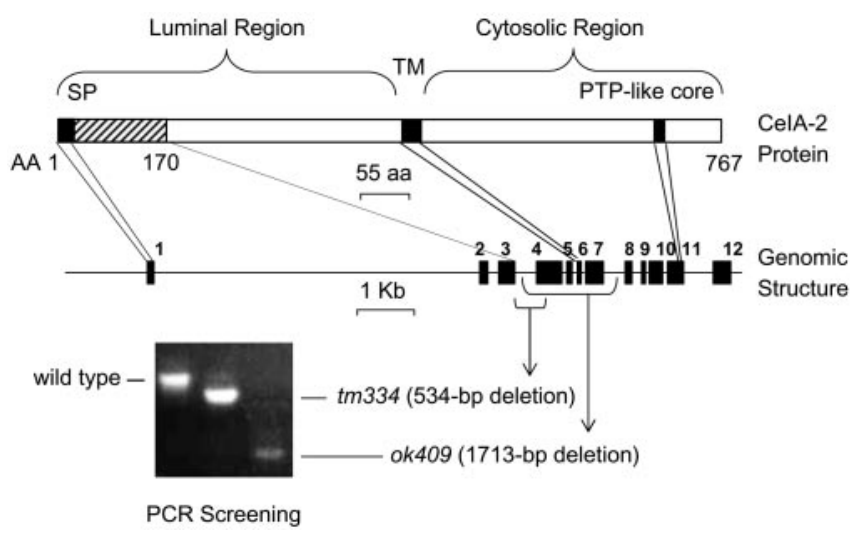

Figure 2. Characterization of ida- 1 deletion alleles. The top diagram represents the CelA-2 protein with certain domains marked relative to their corresponding exons: signal peptide (SP), transmembrane domain (TM), and protein-tyrosine phosphatase-like core (PTP). The bottom diagram shows the genome structure of the ida-1 gene, which consists of 12 exons (filled boxes). The approximate locations of the deleted regions for the alleles ok409 and tm334 are indicated by brackets below the exons. Both deletions result in a premature stop codon attributable to a frame shift after the deletion break points, and each would lack all protein encoded downstream of exon 3, including the TM and PTP domains. The predicted truncated protein product resulting from either deletion is indicated at the $\mathrm{N}$-terminal end by hatched lines. Single-animal PCR products from the ida-1 gene of wild-type animals and homozygous mutants are shown.

1(ok409) and ida-1(tm334) animals had slightly reduced brood sizes of 70 and $90 \%$, respectively, compared with wild-type animals. Because both alleles are predicted nulls, the more severe brood size reduction observed for ok409 may be attributable to strain background differences between the two alleles. We were able to detect two subtle motor program defects in ida-1 mutant animals affecting locomotion and defecation. We compared the mean locomotion rates of wild-type and the two ida-1 mutant alleles by counting body bends of the animals (Miller et al., 1999). The locomotion rate of the mutants was slower than that of the wild type and became progressively worse with age (Table 1). The locomotion rate of $i d a-1$ mutants was $70 \%$ of that of wild type for day 1 adults, $50 \%$ of that of wild type for day 2 adults, and $25 \%$ of that of wild type for day 3 adults. Both ida-1(ok409) and ida1(tm334) mutants also had a minor defecation motor program defect affecting the muscle contractions leading to the expulsion of waste. In wild-type animals, defecation cycles have been well characterized, and each cycle ends with the near simultaneous contraction of the posterior body wall and enteric muscles of the animal, resulting in an expulsion (Thomas, 1990). An examination of defecation in ida-1 mutants revealed that the expulsion step of the cycle was missing in $\sim 10 \%$ of the defecation cycles (data not shown). The subtle motor program defects observed in ida-1(ok409) and ida-1(tm334) mutants was consistent with a mild and general problem in nervous system function.

\section{Loss of CeIA-2 results in impaired synaptic transmission}

The neuronal expression of $i d a-1$ and the minor motor defects of ida-1 mutants led us to investigate synaptic transmission in ida-1 mutant animals. Aldicarb, an acetylcholinesterase inhibitor, has been commonly used to assess acetylcholine release and presynaptic function. To test whether ida-1 mutants have defects in synaptic transmission, we used two different aldicarb assays. First, we used a short-term assay in which we measured the rate of paralysis induced by treatment of adult animals with $0.5 \mathrm{~mm}$ aldicarb for $4 \mathrm{hr}$ at room temperature. Second, we used a growth rate assay in which we counted the number of progeny from parent animals after $96 \mathrm{hr}$ of growth on plates containing various concentrations of aldicarb (Miller et al., 1999). For both assays, ida-1 mutants were compared with wild-type animals and two mutants shown previously to have an aldicarb resistance phenotype, unc-31 (encoding the CAPS homolog) and unc-64 (encoding the syntaxin homolog).

In the short-term aldicarb test, $97 \%$ of wild-type animals were paralyzed after $4 \mathrm{hr}$ on plates containing $0.5 \mathrm{~mm}$ aldicarb. In contrast, only $50 \%$ of ida-1(ok409 or tm334) mutants were paralyzed in the same assay. This compared with $25 \%$ paralysis for unc-31(e169) and only 5\% paralysis for unc-64(e246). In the aldicarb growth rate assay, ida-1(ok409 or tm334) mutants tolerated moderately higher concentrations of aldicarb than did wildtype animals. This is reflected by an increased population rate for ida-1(ok409 or tm334) compared with wild type at all aldicarb concentrations up to $200 \mu \mathrm{M}$ (Fig. 3A). In comparison, unc$31(e 169)$ and $u n c-64(e 246)$ tolerated even higher concentrations of aldicarb than did ida-1(ok409 or tm334). These results demonstrated that ida-1 mutants are aldicarb-resistant; that is, they have reduced acetylcholine release compared with wild-type animals. The aldicarb resistance of ida-1 mutants is modest and at the lower end of the hierarchy of tested mutant strains: wild type $<$ ida-1(ok409 or tm334) < unc-31(e169) < unc-64(e246). The aldicarb effects of the ida-1(ok409 or tm334) mutants appeared to be attributable to neuronal events because levamisole, an agonist of postsynaptic acetylcholine receptors, failed to distinguish between wild-type and ida-1 mutant animals (data not shown).

To confirm that the aldicarb resistance was caused by the ida-1 deletions, we attempted to rescue the defect with either the integrated, full-length ida-1 cDNA fused to GFP ( $\left.\mathrm{P}_{i d a-1} \mathrm{CeIA}-2:: \mathrm{GFP}\right)$ or the cosmid B0244 containing the wild-type ida-1 gene. We found that both DNA constructs reduced the aldicarb resistance of ida-1(ok409) mutants (Fig. 3B). The cosmid B0244 gave full rescue of aldicarb resistance and actually showed enhanced sensitivity to the drug. This mild yet reproducible enhanced sensitivity to aldicarb might have been attributable to overexpression of $i d a-1$ from the cosmid. The $\mathrm{P}_{i d a-1}$ CeIA-2::GFP construct rescue was partial and may have reflected a limited expression profile of this reporter gene (described above) compared with the endogenous ida-1 gene, the mosaic nature or dose of reporter gene expression, or a combination of these factors.

Aldicarb resistance has been commonly attributed to decreased acetylcholine release, presumably from SVs of motor neurons (Miller et al., 1996). We were interested to test whether the mild aldicarb resistance of $i d a-1$ mutants could be rescued by expression of the CeIA-2 cDNA in the cholinergic motor neurons. We made transgenic strains harboring the CeIA-2::GFP fusion protein under the control of a $3.2 \mathrm{~kb}$ promoter region of the acr-2 gene. This promoter has been shown to be specifically expressed in the Aand B-type cholinergic motor neurons of the ventral nerve cord (Nurrish et al., 1999), and that pattern was observed in our transgenic lines (data not shown). $\mathrm{P}_{\mathrm{acr}-2}$ CeIA-2::GFP transgenic strains showed absolutely no rescue of the ida-1(ok409) aldicarb resistance phenotype, using either short or long-term assays (data not shown). This result suggested that loss of CeIA-2 activity in cholinergic motor neurons is not the cause of the aldicarb resistance.

\section{Loss of CeIA-2 does not affect the axonal distribution of SV membrane-associated proteins}

To determine how ida-1 mutations impair presynaptic activity, we examined ida-1 mutants using antibodies specific for two SV membrane-associated proteins, SNT-1 (synaptotagmin; Nonet et 
Table 1. Analysis of behaviors of ida-1 mutants

\begin{tabular}{|c|c|c|c|c|c|c|}
\hline \multirow[b]{2}{*}{ Strain } & \multicolumn{3}{|c|}{ Body bends (times/min) $(n=10)$} & \multirow[b]{2}{*}{ Expulsion $(\%)(n=20)$} & \multirow[b]{2}{*}{ Brood size $(n=10)$} & \multirow[b]{2}{*}{ Life span (d) $(n=25)$} \\
\hline & Day 1 & Day 2 & Day 3 & & & \\
\hline Wild type & $17.0 \pm 4.8$ & $14.9 \pm 4.1$ & $12.7 \pm 2.8$ & $99 \pm 1$ & $290 \pm 30$ & $23 \pm 4$ \\
\hline ok409 & $11.6 \pm 6.5^{*}$ & $7.3 \pm 6.2^{* *}$ & $3.6 \pm 2.1^{* *}$ & $80 \pm 5^{*}$ & $185 \pm 25^{*}$ & $16 \pm 5^{*}$ \\
\hline $\operatorname{tm} 334$ & $10.6 \pm 3.7^{*}$ & $6.4 \pm 2.8^{* *}$ & $3.3 \pm 1.8^{* *}$ & $85 \pm 5^{*}$ & $222 \pm 27^{*}$ & $21 \pm 6$ \\
\hline
\end{tabular}

Values are mean $\pm S E$

${ }^{*}, *$ Statistically significant differences between wild type and mutants $\left({ }^{*} p<0.05,{ }^{* *} p<0.01\right)$.

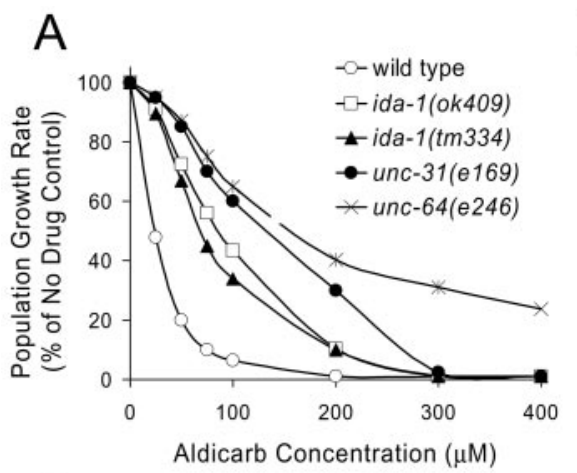

C

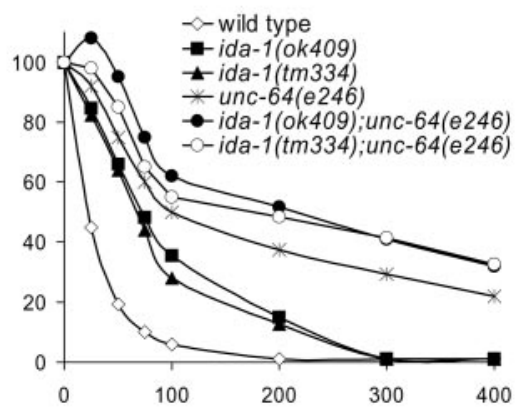

B

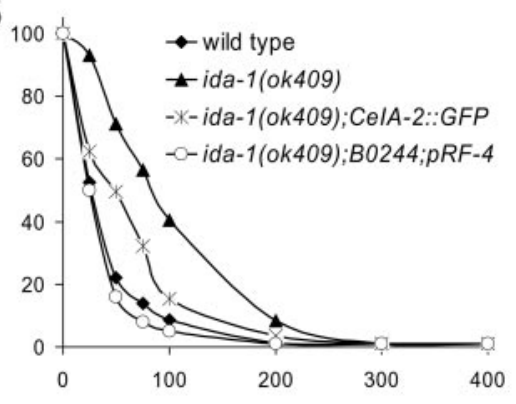

D

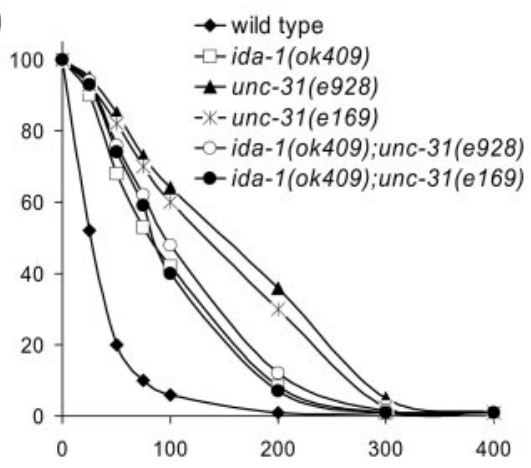

Figure 3. Aldicarb resistance of ida-1 mutants compared with other strains using a growth assay (see Materials and Methods). The population growth rate is shown on the $y$-axis, and the aldicarb concentration is shown on the $x$-axis in all figures. A $100 \%$ growth rate is defined as the number of progeny produced from a starting population of L1 larvae over a $96 \mathrm{hr}$ period in the absence of aldicarb. Curves are representative of the average of duplicate experiments. $A$, The aldicarb resistance of ida-1(ok409 or tm334) is compared with that of unc-31(e169) or unc-64(e246). B, The aldicarb resistance of ida-1(ok409) is partially rescued by expression of the wild-type ida-1 CDNA (CelA-2::GFP). Rescue data shown for the CDNA construct from two independent transgenic lines harboring the CDNA clone are combined. The aldicarb phenotype was fully reversed in transgenic animals harboring the wild-type ida-1 gene on the cosmid B0244. The control is an ida-1(ok409) strain harboring the marker plasmid pRF4 (rol-6) used for generating transgenic lines that confers a Rol phenotype. C, The aldicarb resistance of unc-64(e246) is enhanced by ida-1(ok409 or tm334). D, The aldicarb resistance of both the hypomorphic unc-31(e169) and putative null unc-31(e928) alleles is suppressed by ida-1(ok409 or tm334).

al., 1993) and RAB-3 (Iwasaki et al., 1997), kindly provided by the Nonet and Iwasaki laboratories, respectively (Fig. 4). In wild-type animals (Fig. 4A) and ida-1 mutants (Fig. 4B), anti-SNT-1 antiserum stained only the synapse-rich axons and did not stain cell bodies, suggesting that SNT-1 is correctly localized to synaptic terminals in the ida- 1 mutants. Anti-RAB- 3 antiserum stained predominantly axons in wild-type animals (data not shown) and ida-1 mutants (Fig. 4C), although faint staining of occasional cell bodies was visible. As a control for our immunolocalization of RAB-3, we used the aex-3(sa5) mutant (Fig. 4E) and replicated the previously reported accumulation of RAB-3 in cell bodies of neurons (Iwasaki et al., 1997). These results suggested that there was no gross abnormality in SV vesicle distribution in ida-1 mutants.

It was possible that the bundled axons of the ventral nerve cord were preventing us from noticing, by antibody staining, more subtle aspects of SV distribution in ida-1 mutants. To visu-

alize the distribution of these vesicles at the single-cell level, we used an SNB (synaptobrevin) GFP reporter driven by the str-3 promoter, kindly provided by the laboratory of C. Bargmann (University of California, San Francisco, CA). This reporter gene is expressed in only the two ASI neurons in the head and GFP puncta reflect the accumulation of vesicles in the seven to nine synapses of each axon (Crump et al., 2001). Introduction of this reporter gene into the ida-1(ok409 or tm334) mutant background failed to reveal any SV localization defects (Fig. 4G). Together, these results suggest that $i d a-1$ is not essential for proper transport or localization of SVs in axons, at least at the level of resolution provided by these assays.

As a complement to these studies, we asked whether the neuronal cell body and punctate axonal pattern of the $\mathrm{P}_{\text {ida-1 }}$ CeIA-2::GFP reporter gene was altered in any one of several mutants affecting either vesicle transport (UNC-104/kinesin) or cargo release (UNC-31/CAPS, UNC-64/Syntaxin, and UNC-18/Munc18). We crossed our $\mathrm{P}_{\text {ida-1 }}$ CeIA-2::GFP strain into unc-31(e169), unc-64(e246), unc-18(e81), and unc-104(e1265) mutants and observed the reporter gene expression pattern. In all cases except unc-104(e1265), reporter gene product distribution within individual neurons was indistinguishable from that observed in wild-type animals (data not shown). For unc-104(e1265), we observed very faint cell body staining of a small number of neurons in the head that are not visible in a wild-type background (data not shown). Neurons that strongly express the reporter gene showed no detectable difference in the distribution of the GFP signal within individual neurons. We interpreted the faint GFP signals in unc-104(e1265) animals harboring the $\mathrm{P}_{\text {ida-1 }}$ CeIA-2::GFP reporter gene as an accumulation of normally weak GFP in the cell body of neurons. In a wild-type background, this low-level GFP is undetectable because of its distribution along the axons. This suggested that UNC-104/kinesin might play a minor role in the axonal distribution of the $\mathrm{P}_{\text {ida-1 }}$ CeIA-2::GFP reporter product.

\section{A specific and novel interaction between ida-1 and unc-31/CAPS}

To better understand the function of CeIA-2, we explored possible genetic interactions between mutants in ida-1(ok409 or 
tm334) and several genes encoding factors involved in neurosecretory vesicle trafficking and cargo release. This included genes affecting SV docking and fusion (unc-18, rab-3, and unc-64/syntaxin), the RAB-3 nucleotide exchange factor AEX-3 (aex-3), and the DCV fusion factor UNC-31/CAPS (unc-31). In addition to the distinctive phenotypes of each mutant, they are all aldicarb-resistant. Therefore, we assayed aldicarb resistance of each of these mutants alone and in combination with the ida- 1 alleles. Of the tested mutants, only unc-31/ CAPS and unc-64/syntaxin had detectable genetic interactions with ida-1(ok409 or tm334). Double mutants between unc64(e246) and ida-1 (ok409 or tm334) had an enhanced aldicarb resistance phenotype (Fig. 3C). No other double mutant showed enhanced aldicarb resistance (data not shown), although aex-3(sa5) mutants alone have such high resistance to aldicarb that enhancement might not be detected in these assays. In contrast, double mutants between unc-31(e169) and ida-1(ok409 or tm334) had an aldicarb resistance phenotype that was indistinguishable from that of ida1(ok409 or tm334) alone; that is, ida-1 was epistatic to $u n c-31$ (Fig. 3D). To confirm this genetic interaction, we made double mutants between ida-1(ok409) and the putative null deletion allele unc31(e928). As with the previously tested hypomorphic allele, ida1(ok409) was epistatic to unc-31(e928) for aldicarb resistance (Fig. 3D). Loss of ida-1 did not suppress the overt Unc phenotype of either unc-31 allele, although the body bend assay showed a slight improvement in double mutant combinations (data not shown). These results demonstrated that $i d a-1$ acts genetically in an $u n c-31$ pathway, suggesting a role for CeIA-2 in DCV trafficking, cargo release, or both.

A distinctive phenotype of $u n c-31$ mutants is the accumulation of serotonin in the cell bodies of serotonergic neurons (Desai et al., 1988), presumably reflecting the direct effect of impaired release of this DCV-specific neurotransmitter. To determine whether ida-1 mutants affected serotonin release, either alone or in combination with unc-31 mutants, we stained mutants with anti-serotonin antibodies. As previously reported (Desai et al., 1988), there is an easily detectable increase of serotonin staining in the head neurons of unc-31 mutants. For ida-1 mutants, we focused on the serotonin-containing neurons having high levels of $\mathrm{P}_{i d a-1}$ CeIA-2::GFP reporter gene expression, namely, the HSN neurons and uv1 cells. The serotonin staining results for these cells in ida-1 mutants were clearly not as robust as observed for the head neurons of unc-31 mutant animals. Although there was a tendency for ida-1 mutants to have higher levels of serotonin staining in the HSN and uv1 cells, the assays were not quantitative, and the variability from animal to animal made it difficult to draw any conclusion. Staining of ida-1; unc-31 double mutants revealed the same increase in serotonin levels in the head neurons as observed for $u n c-31$ mutants alone.

ida-1 interacts genetically with genes in the dauer pathway Under ideal conditions, the C. elegans life cycle comprises embryogenesis, four larval stages (L1-L4), and adulthood. However, in response to overcrowding, starvation, or elevated temperatures, an alternative life cycle that is suited to dispersal is adopted in which L2 larvae enter diapause and become dauer larvae. On sensing improved conditions, the dauer larva exits diapause, molting into the L4 stage to resume the life cycle. At least three genetically distinct pathways regulate dauer formation in C. elegans (Kimura et al., 1997; Patterson and Padgett, 2000). Two of these pathways, transforming growth factor $\beta$ (TGF- $\beta$ )-like and cGMP, act through a downstream nuclear hormone receptor encoded by daf-12. The third pathway is an insulin-like signaling pathway that acts through the forkhead-related factor encoded by daf-16. Several of the insulin-like pathway factors also interact genetically with daf-12, suggesting that the dauer decision reflects a complex integration of signaling from multiple inputs in a variety of cells.

Previous studies have demonstrated that $u n c-31$ and $u n c-64$ share a common dauer-related phenotype, namely, that both mutations result in an enhanced rate of dauer formation at elevated temperatures (Iwasaki et al., 1997). These genes have been placed genetically in the insulin-like signaling pathway for dauer formation (Ailion et al., 1999). We tested whether ida-1 (ok409 or tm334) mutants, either alone or in combination with $u n c-31$ and unc-64 mutants, had a dauer phenotype. We let animals lay embryos for 2-3 hr and then shifted those embryos to an elevated temperature and scored the number of dauer larvae formed after $3 \mathrm{~d}$. As observed by others previously, the exact temperature is critical in determining the percentage of animals that form dauers (Ailion and Thomas, 2000), and care was taken to precisely measure the temperature of plates within the incubators.

At $26^{\circ} \mathrm{C}$, wild-type and ida-1 (ok409 or tm334) animals rarely, if ever, formed dauers. In contrast, both unc-31(e169) and unc$64(e 246)$ mutants formed dauers at $26^{\circ} \mathrm{C}$ with rates of 38 and $35 \%$, respectively, in our assays. Double mutants between unc- 


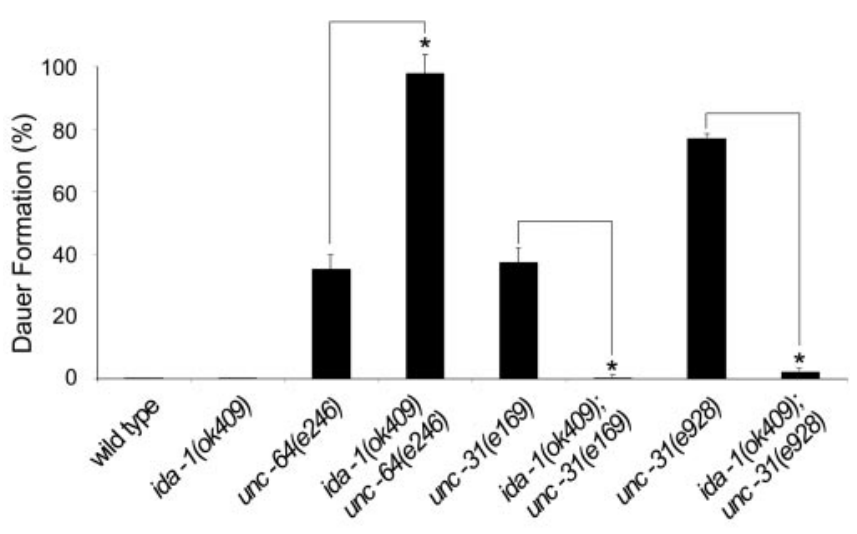

Figure 5. Effect of ida- 1 mutants on the percentage of dauer formation of unc- 64 and unc-31 mutants. At $26^{\circ} \mathrm{C}$, wild-type and ida-1(ok409) animals show no dauer formation, whereas $\sim 35 \%$ of unc-64(e246) animals form dauers. Dauer formation of the unc-64(e246) strain was enhanced to $>92 \%$ in the double mutants with ida- 1 (ok 409$)$. At $26^{\circ} \mathrm{C}, \sim 37 \%$ of unc-37(e 169$)$ or $80 \%$ of unc-37 (e928) animals form dauers. Dauer formation was dramatically suppressed in double mutants with ida-1(ok409) and either the hypomorphic unc-31(e169) allele or the putative null allele unc-37(e928). Similar results were observed for ida-1(tm334) (data not shown). Values that differ significantly are indicated $\left({ }^{*} p<0.01\right)$.

64(e246) and ida-1(ok409 or tm334) showed dramatic enhancement of the dauer phenotype at $26^{\circ} \mathrm{C}$, producing between 90 and $\sim 98 \%$ dauers (Fig. 5). The opposite effect was observed in unc31 (e169); ida-1 (ok409 or tm334) double mutants. The dauer phenotype of $u n c-31$ (e169) was completely suppressed in the ida-1 mutant background; no dauers were formed by the doublemutant animals at $26^{\circ} \mathrm{C}$ (Fig. 5). Because the unc-31(e169) mutant is a hypomorphic allele, loss of dauer formation in the ida1(ok409 or tm334); unc-31(e169) double mutants might have reflected an increase in UNC-31 activity in the absence of CeIA-2. To test this genetically, we made double mutants between ida1 (ok409 or tm334) and the putative null unc-31(e928) allele. As observed with the hypomorphic unc-31 allele, unc-31(e928); ida1 ( ok409 or tm334) double mutants dramatically reduced the percentage of dauers $(<5 \%)$ formed at $26^{\circ} \mathrm{C}$ (Fig. 5). These results demonstrated that a low level of UNC-31 activity is not the underlying cause of the suppression of dauer formation in ida-1; unc-31 double mutants. The ability of $u n c-31$ to affect dauer formation is dependent on ida-1 activity.

We were interested to know whether the effects of ida-1 mutations on dauer formation were restricted to the insulin-like signaling pathway. We therefore made double-mutant combinations between ida-1(ok409) and mutants of genes affecting other branches of the genetic pathway for dauer formation. The three genes we tested were daf-7, daf-11, and daf-12. The daf-7 gene encodes a member of the TGF- $\beta$ superfamily (Ren et al., 1996; Schackwitz et al., 1996). Double mutants between the daf-7 temperature-sensitive loss-of-function allele e1372 and ida1 (ok409) resulted in a slight enhancement of dauer formation at $22.5^{\circ} \mathrm{C}(40 \% ; n=105)$ compared with daf-7(e1372) alone (28\%; $n=120)$. The daf-11 gene encodes a guanylate cyclase, and the $m 47$ allele is a predicted null (Birnby et al., 2000). The ida1(ok409) daf-11(m47) double mutants also showed a slight enhancement of dauer formation at $25^{\circ} \mathrm{C}(92 \% ; n=147)$ compared with daf-11(m47) alone $(80 \% ; n=134)$. To test interactions between ida-1 and daf-12, we used a constitutive dauer (daf-c) allele, rh273. There was no significant difference in the rate of dauer formation at any temperature tested in ida-1(ok409); daf12 (rh273) double mutants $(38 \% ; n=133)$ compared with daf-

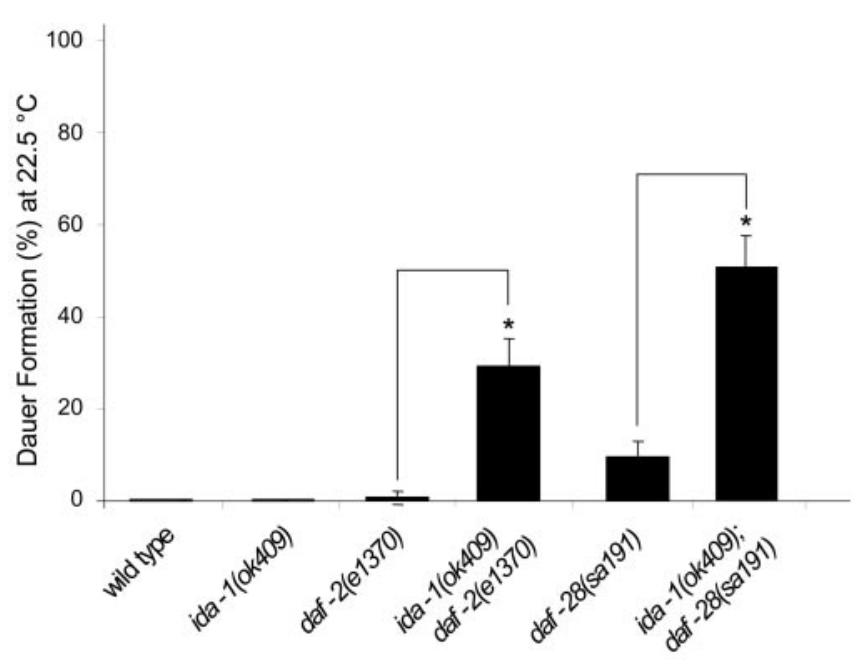

Figure 6. Effect of ida-1 mutants on the percentage of dauer formation of daf- 2 and daf-28 mutants. At $22.5^{\circ} \mathrm{C}$, wild-type, ida-1(ok409), and daf-2(e1370) animals show almost no dauer formation; $10 \%$ of daf-28(sa191) form dauers. Both ida-1(ok409) and ida-1(tm334) (data not shown) mutants significantly enhance either daf-2(e1370) or daf-28(sa191) dauer formation. Values that differ significantly are indicated $\left({ }^{*} p<0.01\right)$.

$12(r h 273)$ alone $(40 \% ; n=186)$. Interpretation of the daf-12 interaction results is complicated by the difficulty in scoring the partial and transient dauers generated by this daf-12 allele and the complex genetic interactions between $d a f-12$ and all branches of the genetic pathway of dauer formation. The results from ida-1 double mutants with daf-7 and daf-11 are consistent with, but do not prove, a role for $i d a-1$ that is predominantly restricted to the insulin-like signaling branch of the dauer formation pathway.

\section{ida-1 alleles enhance weak alleles of multiple genes in the insulin-like signaling pathway}

Many of the components of the C. elegans insulin-like signaling pathway have been characterized, and there are numerous parallels to insulin signaling in mammals. In C. elegans, the binding of ligand (e.g., DAF-28) to the insulin receptor-like factor encoded by the daf-2 gene has been shown to act through phosphatidylinositol (PI) 3-kinase (age-1), Akt/PKBs (akt-1 and akt-2), phosphoinositide-dependent kinases ( $p d k-1$ and $p d k-2)$, and other factors to regulate the action of the forkhead transcription factor DAF-16 (daf-16), which, in turn, regulates dauer formation (Paradis and Ruvkun, 1998; Paradis et al., 1999; Pierce et al., 2001; Li et al., 2003). The genetic interactions we found between ida-1 and two genes, unc-31 and unc-64, in the insulin-like branch of the dauer pathway prompted us to assay additional genes in this branch. We made double mutants between ida$1(t m 334$ or $o k 409)$ and a daf-2 allele (e1370) or a daf-28 allele (sa191) and assayed the percentage of dauers formed at $22.5^{\circ} \mathrm{C}$ compared with each mutant alone. The daf-2(e1370) allele is hypomorphic, whereas daf-28(sa191) appears to be antimorphic ( $\mathrm{Li}$ et al., 2003). At $22.5^{\circ} \mathrm{C}$, wild-type animals and ida-1(tm334 or ok409) mutants formed no dauers, whereas daf-2(e1370) mutants formed $0.5 \%$ dauers, and daf-28(sa191) mutants formed $10 \%$ dauers (Fig. 6). In comparison, ida-1(tm334 or ok409) daf2(e1370) double mutants had an enhanced rate of dauer formation, with a level of $30 \%$. Similarly, the ida-1(tm334 or ok409); daf-28(sa191) double mutants had an enhanced rate (e.g., 50 vs $10 \%)$ of dauer formation compared with either single mutant (Fig. 6). These results are consistent with a role of CeIA-2 in the insulin-like signaling branch of the dauer regulation pathway. 


\section{Discussion}

\section{CeIA-2 genetically interacts with UNC-31/CAPS}

IA-2 is a transmembrane protein-tyrosine phosphatase implicated in hormone neurosecretion. We find that a $C$. elegans IA-2::GFP translational reporter gene is present in both the cell body of neurons and in a punctate axonal distribution that correlates with a synaptic marker. We have begun to genetically dissect the role of CeIA-2 in neurosecretion during C. elegans development using putative null alleles of $i d a-1$, the gene encoding CeIA-2. ida-1 mutants have subtle motor program defects and a mild aldicarb resistance. Double mutant combinations between ida-1 and previously characterized genes involved in SV or DCV exocytosis suggest a specific role for CeIA-2 in DCV cargo release.

The most surprising result of our studies was the epistatic relationship between $i d a-1$ and $u n c-31$ mutants. Both the aldicarb resistance and dauer phenotypes of $u n c-31$ mutants are dependent on the presence of wild type ida-1 activity. To our knowledge, ida-1 is the first gene described capable of suppressing these unc-31 mutant phenotypes. This genetic interaction suggests that CeIA-2 modulates, directly or indirectly, the activity of UNC-31/CAPS.

CAPS is one of the rare factors suggested to function specifically in DCV exocytosis. CAPS appears to be able to interact with both the plasma membrane and DCVs, suggesting it may physically bridge these two substrates. Recent reports demonstrated that mammalian CAPS associates with phosphatidylinositol 4,5bisphosphate $[\mathrm{PI}(4,5) \mathrm{P} 2]$ on the plasma membrane via its pleckstrin homology domain and also targets to DCVs via its distal C-terminal sequences (Martin and Kowalchyk, 1997; Grishanin et al., 2002). After docking of DCVs to the plasma membrane, the synthesis of PI(4,5)P2 and PI 4-phosphate induced by PI 4-kinases and PI 5-kinases are thought to account for at least part of the requirement for Mg-ATP in the priming process (Hay et al., 1995; Wiedemann et al., 1996; Olsen et al., 2003). Therefore, interactions with both DCVs and the plasma membrane may play an important role in the mechanism by which CAPS facilitates the release of DCV cargo. The binding determinants of CAPS on DCVs have not been identified. Given our results showing a genetic interaction between $u n c-31$ and $i d a-1$, it would be interesting to examine whether the PTP domain-containing cytosolic region of CeIA-2 interacts with the distal $C$-terminal sequences of UNC-31/CAPS or with a common effector molecule.

Studies of Drosophila CAPS provide further support that this factor is a critical and specific regulator of DCV exocytosis. In Drosophila, null dcaps mutants have severe movement defects and die late in embryogenesis (Renden et al., 2001). Examination of synaptic terminals revealed a greater than threefold increase in DCVs in these mutants suggesting an essential function for CAPS in vesicle fusion and cargo release. Importantly, these studies also demonstrated an accumulation of SVs at neuromuscular junctions in dcaps mutants (Renden et al., 2001; Richmond and Broadie, 2002). This SV phenotype was shown to be non-cellautonomous and secondary to an upstream defect, most likely impaired release of DCV cargo in neurosecretory cells modulating the activity of motor neurons.

These observations in Drosophila provide an excellent framework in which to interpret our own results with ida-1 in C. elegans. ida-1 mutant alleles have a mild aldicarb resistance phenotype, suggesting a deficit in acetylcholine release from SVs. As in Drosophila, we suggest that the aldicarb resistance of ida-1 null mutants reflects a secondary consequence of impaired DCV function in upstream neurons. This conclusion is consistent with our inability to rescue the aldicarb phenotype of ida-1(ok409) when expression of a rescuing transgene is restricted to the A- and B-type cholinergic motor neurons. Three other mutants of putative DCV-specific proteins in C. elegans have reduced acetylcholine release, resulting in an aldicarb resistance phenotype. These are UNC-31/CAPS (Iwasaki et al., 1997; Berwin et al., 1998; Renden et al., 2001; Munoz and Riddle, 2003) and two peptide hormone-processing enzymes, egg laying-defective (EGL)-21/ carboxypeptidase E and EGL-3/proprotein convertase 2 (Jacob and Kaplan, 2003). It seems likely that impaired DCV cargo release in upstream neurosecretory cells leads to aldicarb resistance in all of these mutants. The molecular mechanism(s) underlying DCV cargo release and its modulation by CeIA-2 and UNC-31/ CAPS is not revealed by our genetic studies. However, our studies suggest that understanding the molecular relationship between CeIA-2 and UNC-31/CAPS will be critical to understanding fully the mechanism of DCV exocytosis.

\section{CeIA-2 and the regulation of insulin-like signaling in C. elegans}

Dauer formation is regulated by at least three pathways in $C$. elegans: insulin-like signaling, TGF- $\beta$-like signaling, and cGMP. These pathways have been well studied genetically allowing us to explore interactions between $i d a-1$ and dauer pathway components. We find that ida-1 shows the strongest genetic interactions with mutants in the insulin-like signaling pathway. This includes the genes encoding UNC-31/CAPS, UNC-64/syntaxin, the insulin-like receptor DAF-2, and insulin-like ligand DAF-28 (Ailion et al., 1999; Li et al., 2003). The ability of $i d a-1$ mutants to enhance, albeit weakly, loss-of-function alleles in the TGF- $\beta$ (daf-7) and cGMP (daf-11) branches of the dauer pathway is also consistent with a role for CeIA-2 that is primarily restricted to insulin-like signaling. Although ida-1 mutants do not have a dauer phenotype on their own, CeIA-2 appears to be a modifier of processes regulated by genes in the insulin-like signaling pathway of dauer formation.

Our results suggesting that CeIA-2 functions in the C. elegans insulin-like signaling pathway are strikingly similar to those described for IA-2 function in mammals. Mouse knock-outs of the gene encoding IA-2 result in elevated blood glucose levels in glucose tolerance tests (Saeki et al., 2002). Moreover, cultured pancreatic $\beta$ cells from these knock-out mice have a $40-45 \%$ reduction in insulin release after glucose stimulation. In contrast to the single IA-2 family member found in invertebrates, mice have two related genes, IA- 2 and IA- $2 \beta$ (phogrin). In mice, neither knockout alone nor in combination eliminates insulin release (J. $\mathrm{Ku}-$ bosaki and A. L. Notkins, unpublished data). As in the worm, then, it appears that neither IA- 2 nor IA- $2 \beta$ is essential for insulin secretion, and, although impaired under stressful conditions, limited insulin signaling can occur in mammals and C. elegans in the complete absence of IA-2/IA- $2 \beta$ and CeIA-2, respectively.

The identification of CeIA-2 as a component involved in DCV cargo release adds to our general understanding of DCV trafficking. Future studies will be needed to transform our genetic results into molecular detail. For example, what factors interact directly with CeIA-2, and what is the molecular mechanism by which CeIA-2 facilitates DCV cargo release? Exploitation of C. elegans genetics will be an important adjunct to these future studies because it will allow us to identify additional, and perhaps novel, components regulating DCV cargo release.

\section{References}

Ailion M, Thomas JH (2000) Dauer formation induced by high temperatures in Caenorhabditis elegans. Genetics 156:1047-1067. 
Ailion M, Inoue T, Weaver CI, Holdcraft RW, Thomas JH (1999) Neurosecretory control of aging in Caenorhabditis elegans. Proc Natl Acad Sci USA 96:7394-7397.

Berghs S, Aggujaro D, Dirkx R Jr, Maksimova E, Stabach P, Hermel JM, Zhang JP, Philbrick W, Slepnev V, Ort T, Solimena M (2000) betaIV spectrin, a new spectrin localized at axon initial segments and nodes of Ranvier in the central and peripheral nervous system. J Cell Biol 151:985-1002.

Berwin B, Floor E, Martin TF (1998) CAPS (mammalian UNC-31) protein localizes to membranes involved in dense-core vesicle exocytosis. Neuron 21:137-145.

Birnby DA, Link EM, Vowels JJ, Tian H, Colacurcio PL, Thomas JH (2000) A transmembrane guanylyl cyclase (DAF-11) and Hsp90 (DAF-21) regulate a common set of chemosensory behaviors in Caenorhabditis elegans. Genetics 155:85-104.

Cai T, Krause MW, Odenwald WF, Toyama R, Notkins AL (2001) The IA-2 gene family: homologs in Caenorhabditis elegans, Drosophila and zebrafish. Diabetologia 44:81-88.

Chalfie M, Jorgensen EM (1998) C. elegans neuroscience: genetics to genome. Trends Genet 14:506-512.

Crump JG, Zhen M, Jin Y, Bargmann CI (2001) The SAD-1 kinase regulates presynaptic vesicle clustering and axon termination. Neuron 29:115-129.

Desai C, Garriga G, McIntire SL, Horvitz HR (1988) A genetic pathway for the development of the Caenorhabditis elegans HSN motor neurons. Nature 336:638-646.

Egan CR, Chung MA, Allen FL, Heschl MF, Van Buskirk CL, McGhee JD (1995) A gut-to-pharynx/tail switch in embryonic expression of the Caenorhabditis elegans ges-1 gene centers on two GATA sequences. Dev Biol 170:397-419.

Finney M, Ruvkun G (1990) The unc-86 gene product couples cell lineage and cell identity in C. elegans. Cell 63:895-905.

Gerber SH, Sudhof TC (2002) Molecular determinants of regulated exocytosis. Diabetes 51[Suppl 1]:S3-S11.

Grishanin RN, Klenchin VA, Loyet KM, Kowalchyk JA, Ann K, Martin TF (2002) Membrane association domains in Ca2+-dependent activator protein for secretion mediate plasma membrane and dense-core vesicle binding required for $\mathrm{Ca} 2+$-dependent exocytosis. J Biol Chem 277:22025-22034.

Hay JC, Fisette PL, Jenkins GH, Fukami K, Takenawa T, Anderson RA, Martin TF (1995) ATP-dependent inositide phosphorylation required for $\mathrm{Ca}(2+)$-activated secretion. Nature 374:173-177.

Iwasaki K, Toyonaga R (2000) The Rab3 GDP/GTP exchange factor homolog AEX-3 has a dual function in synaptic transmission. EMBO J 19:4806-4816.

Iwasaki K, Staunton J, Saifee O, Nonet M, Thomas JH (1997) aex-3 encodes a novel regulator of presynaptic activity in C. elegans. Neuron 18:613-622.

Jacob TC, Kaplan JM (2003) The EGL-21 carboxypeptidase E facilitates acetylcholine release at Caenorhabditis elegans neuromuscular junctions. J Neurosci 23:2122-2130.

Kawasaki E, Hutton JC, Eisenbarth GS (1996) Molecular cloning and characterization of the human transmembrane protein tyrosine phosphatase homologue, phogrin, an autoantigen of type 1 diabetes. Biochem Biophys Res Commun 227:440-447.

Kimura KD, Tissenbaum HA, Liu Y, Ruvkun G (1997) daf-2, an insulin receptor-like gene that regulates longevity and diapause in Caenorhabditis elegans. Science 277:942-946.

Lan MS, Lu J, Goto Y, Notkins AL (1994) Molecular cloning and identification of a receptor-type protein tyrosine phosphatase, IA-2, from human insulinoma. DNA Cell Biol 13:505-514.

Lan MS, Wasserfall C, Maclaren NK, Notkins AL (1996) IA-2, a transmembrane protein of the protein tyrosine phosphatase family, is a major autoantigen in insulin-dependent diabetes mellitus. Proc Natl Acad Sci USA 93:6367-6370.

Li W, Kennedy SG, Ruvkun G (2003) daf-28 encodes a C. elegans insulin superfamily member that is regulated by environmental cues and acts in the DAF-2 signaling pathway. Genes Dev 17:844-858.

Loer CM, Kenyon CJ (1993) Serotonin-deficient mutants and male mating behavior in the nematode Caenorhabditis elegans. J Neurosci 13:5407-5417.

Lu J, Li Q, Xie H, Chen ZJ, Borovitskaya AE, Maclaren NK, Notkins AL, Lan MS (1996) Identification of a second transmembrane protein tyrosine phosphatase, IA-2beta, as an autoantigen in insulin-dependent diabetes mellitus: precursor of the $37-\mathrm{kDa}$ tryptic fragment. Proc Natl Acad Sci USA 93:2307-2311.
Magistrelli G, Toma S, Isacchi A (1996) Substitution of two variant residues in the protein tyrosine phosphatase-like PTP35/IA-2 sequence reconstitutes catalytic activity. Biochem Biophys Res Commun 227:581-588.

Martin TF, Kowalchyk JA (1997) Docked secretory vesicles undergo Ca2+activated exocytosis in a cell-free system. J Biol Chem 272:14447-14453.

Mello C, Fire A (1995) DNA transformation. Methods Cell Biol 48:451-482.

Miller KG, Alfonso A, Nguyen M, Crowell JA, Johnson CD, Rand JB (1996) A genetic selection for Caenorhabditis elegans synaptic transmission mutants. Proc Natl Acad Sci USA 93:12593-12598.

Miller KG, Emerson MD, Rand JB (1999) Goalpha and diacylglycerol kinase negatively regulate the Gqalpha pathway in C. elegans. Neuron 24:323-333.

Munoz MJ, Riddle DL (2003) Positive selection of Caenorhabditis elegans mutants with increased stress resistance and longevity. Genetics 163:171-180.

Nguyen M, Alfonso A, Johnson CD, Rand JB (1995) Caenorhabditis elegans mutants resistant to inhibitors of acetylcholinesterase. Genetics 140:527-535.

Nonet ML (1999) Visualization of synaptic specializations in live C. elegans with synaptic vesicle protein-GFP fusions. J Neurosci Methods 89:33-40.

Nonet ML, Grundahl K, Meyer BJ, Rand JB (1993) Synaptic function is impaired but not eliminated in C. elegans mutants lacking synaptotagmin. Cell 73:1291-1305.

Notkins AL (2002) Immunologic and genetic factors in type 1 diabetes. J Biol Chem 277:43545-43548.

Nurrish S, Segalat L, Kaplan JM (1999) Serotonin inhibition of synaptic transmission: Galpha(0) decreases the abundance of UNC-13 at release sites. Neuron 24:231-242.

Olsen HL, Hoy M, Zhang W, Bertorello AM, Bokvist K, Capito K, Efanov AM, Meister B, Thams P, Yang SN, Rorsman P, Berggren PO, Gromada J (2003) Phosphatidylinositol 4-kinase serves as a metabolic sensor and regulates priming of secretory granules in pancreatic beta cells. Proc Natl Acad Sci USA 100:5187-5192.

Ort T, Voronov S, Guo J, Zawalich K, Froehner SC, Zawalich W, Solimena M (2001) Dephosphorylation of beta2-syntrophin and Ca2+/mu-calpainmediated cleavage of ICA512 upon stimulation of insulin secretion. EMBO J 20:4013-4023.

Paradis S, Ruvkun G (1998) Caenorhabditis elegans Akt/PKB transduces insulin receptor-like signals from AGE-1 PI3 kinase to the DAF-16 transcription factor. Genes Dev 12:2488-2498.

Paradis S, Ailion M, Toker A, Thomas JH, Ruvkun G (1999) A PDK1 homolog is necessary and sufficient to transduce AGE-1 PI3 kinase signals that regulate diapause in Caenorhabditis elegans. Genes Dev 13:1438-1452.

Patterson GI, Padgett RW (2000) TGF beta-related pathways: roles in Caenorhabditis elegans development. Trends Genet 16:27-33.

Pierce SB, Costa M, Wisotzkey R, Devadhar S, Homburger SA, Buchman AR, Ferguson KC, Heller J, Platt DM, Pasquinelli AA, Liu LX, Doberstein SK, Ruvkun G (2001) Regulation of DAF-2 receptor signaling by human insulin and ins-1, a member of the unusually large and diverse C. elegans insulin gene family. Genes Dev 15:672-686.

Pouli AE, Karagenc N, Wasmeier C, Hutton JC, Bright N, Arden S, Schofield JG, Rutter GA (1998) A phogrin-aequorin chimaera to image free Ca2+ in the vicinity of secretory granules. Biochem J 330:1399-1404.

Rand JB, Nonet ML (1997) Synaptic transmission. In: C. elegans II (Riddle DL, Blumenthal T, Meyer BJ, Priess JR, eds), pp 611-644. Plainview, NY: Cold Spring Harbor Laboratory.

Ren P, Lim CS, Johnsen R, Albert PS, Pilgrim D, Riddle DL (1996) Control of C. elegans larval development by neuronal expression of a TGF-beta homolog. Science 274:1389-1391.

Renden R, Berwin B, Davis W, Ann K, Chin CT, Kreber R, Ganetzky B, Martin TF, Broadie K (2001) Drosophila CAPS is an essential gene that regulates dense-core vesicle release and synaptic vesicle fusion. Neuron 31:421-437.

Richmond JE, Broadie KS (2002) The synaptic vesicle cycle: exocytosis and endocytosis in Drosophila and C. elegans. Curr Opin Neurobiol 12:499-507.

Saeki K, Zhu M, Kubosaki A, Xie J, Lan MS, Notkins AL (2002) Targeted disruption of the protein tyrosine phosphatase-like molecule IA-2 results in alterations in glucose tolerance tests and insulin secretion. Diabetes 51:1842-1850.

Schackwitz WS, Inoue T, Thomas JH (1996) Chemosensory neurons function in parallel to mediate a pheromone response in C. elegans. Neuron 17:719-728.

Solimena M, Dirkx Jr R, Hermel JM, Pleasic-Williams S, Shapiro JA, Caron L, Rabin DU (1996) ICA 512, an autoantigen of type I diabetes, is an intrinsic membrane protein of neurosecretory granules. EMBO J 15:2102-2114.

Squire MD, Tornoe C, Baylis HA, Fleming JT, Barnard EA, Sattelle DB 
(1995) Molecular cloning and functional co-expression of a Caenorhabditis elegans nicotinic acetylcholine receptor subunit (acr-2). Receptors Channels 3:107-115.

Thomas JH (1990) Genetic analysis of defecation in Caenorhabditis elegans. Genetics 124:855-872.

Walent JH, Porter BW, Martin TF (1992) A novel 145 kd brain cytosolic protein reconstitutes $\mathrm{Ca}(2+)$-regulated secretion in permeable neuroendocrine cells. Cell 70:765-775.

White JG, Southgate E, Thomas JN, Brenner S (1986) The structure of the nervous system of the nematode Caenorhabditis elegans. Philos Trans R Soc Lond B Biol Sci 314:1-340.

Wiedemann C, Schafer T, Burger MM (1996) Chromaffin granule-associated phosphatidylinositol 4-kinase activity is required for stimulated secretion. EMBO J 15:2094-2101.

Williams BD (1995) Genetic mapping with polymorphic sequence-tagged sites. In: Caenorhabditis elegans: modern biological analysis of an organism (Epstein HF, Shakes D, eds), pp 81-96. New York: Academic.

Wishart MJ, Dixon JE (1998) Gathering STYX: phosphatase-like form predicts functions for unique protein-interaction domains. Trends Biochem Sci 23:301-306.

Zahn TR, Macmorris MA, Dong W, Day R, Hutton JC (2001) IDA-1, a Caenorhabditis elegans homolog of the diabetic autoantigens IA-2 and phogrin, is expressed in peptidergic neurons in the worm. J Comp Neurol 429:127-143. 